Microscale Thermophysical Engineering, 8:361-381, 2004

Copyright $(9)$ Taylor \& Francis Inc.

ISSN: 1089-3954 print/1091-7640 online

DOI: $10.1080 / 10893950490516901$

\title{
TRANSIENT BEHAVIOR OF THE VISCOUS MICROPUMP
}

\author{
M. Abdelgawad, I. Hassan, and N. Esmail \\ Department of Mechanical and Industrial Engineering, Concordia University, \\ Montreal, Quebec, Canada
}

In the present study, the transient performance of the viscous micropump will be investigated numerically. The viscous micropump's operation depends mainly on viscous forces and can operate in any situation where viscous forces are dominant. All the micropump calculations are reported in nondimensional quantities, which allows for the prediction of the micropump performance, regardless of the dimensions or the fluid that is used. The effect of the microchannel height, rotor eccentricity, Reynolds number, and pump load on the transient performance of the viscous micropump has been studied in detail. The steady state performance was compared with the available experimental data and was found to be in a very good agreement. The rotor eccentricity was determined to be the parameter that affected the transient performance of the micropump the most significantly. This work provides a foundation for future research on the subject of fluid phenomena in viscous micropumps.

Keywords transient microfluidics, viscous micropump, CFD

\section{INTRODUCTION}

Micropumps are among the most developed of all MEMS devices and have already been implemented into the mainstream. For example, micropumps are used in ink jet printers to inject ink droplets as well as in fuel injector applications. Micropumps operate as a result of completely different principles than those applied in traditional pumps like the axial or centrifugal pumps. Micro dimensions limit the effect of centrifugal forces and inertia forces in general, and the large surface-to-volume ratio amplifies the effect of viscous forces, rendering it the dominant force at the microscale [1]. Positive displacement pumping is the most prevalent method used in micropumps, yet the actuation of the reciprocating diaphragm is achieved by applying different principles. Thermopneumatic, piezoelectrical, and electrostatic actuations are examples of the actuation methods used [2]. The problem with positive displacement micropumps is that they require check valves at the inlet and outlet ports, which adds to the complexity of design, especially on such a small scale. To simplify the design, Stemme and Stemme [3] suggested replacing the check valves with a nozzle at the inlet and a diffuser at the exit, as reported in Erik and Goran [4]. Yet the idea is not practical since, in order to operate properly, certain operating conditions are required, which do not apply at all times.

Received 11 July 2003; accepted 24 October 2003.

Address correspondence to I. Hassan, Department of Mechanical and Industrial Engineering, Concordia University, 1455 de Maisonneuue Blvd. West, H549, Montreal, Quebec H3G 1M8, Canada. E-mail: Hassan@me.concordia.ca 


\begin{tabular}{|c|c|c|c|}
\hline \multicolumn{4}{|c|}{ NOMENCLATURE } \\
\hline$C_{D}$ & cylinder drag coefficient & $S$ & non-dimensional channel height \\
\hline$C_{L}$ & cylinder lift coefficient & $t$ & time $(\mathrm{s})$ \\
\hline$C_{M}$ & cylinder moment coefficient & $t^{*}$ & nondimensional time \\
\hline$d$ & cylinder diameter (m) & $\Delta t$ & time step size (s) \\
\hline$F_{L}$ & lift force on cylinder $(\mathrm{N})$ & $\bar{u}$ & average velocity inside micropump $(\mathrm{m} / \mathrm{s})$ \\
\hline$F_{D}$ & drag force on cylinder $(\mathrm{N})$ & $u^{*}$ & nondimensional average velocity \\
\hline$h$ & channel height (m) & $U$ & cylinder surface velocity $(\mathrm{m} / \mathrm{s})$ \\
\hline$\dot{m}$ & pump mass flow rate $(\mathrm{kg} / \mathrm{s})$ & $y_{c}$ & distance from channel axis to cylinder \\
\hline$M$ & moment on cylinder $(\mathrm{N} \cdot \mathrm{m})$ & & center $(\mathrm{m})$ \\
\hline$P_{\text {in }}$ & pump inlet pressure $(\mathrm{Pa})$ & & \\
\hline$P_{\text {out }}$ & pump outlet pressure $(\mathrm{Pa})$ & \multicolumn{2}{|c|}{ Greek Letters } \\
\hline$P^{*}$ & nondimensional pressure & $\epsilon$ & cylinder eccentricity \\
\hline$\Delta P$ & pump pressure rise $(\mathrm{Pa})$ & $\eta$ & pump efficiency \\
\hline$\Delta P^{*}$ & non-dimensional pressure rise & $v$ & fluid kinematic viscosity $\left(\mathrm{m}^{2} / \mathrm{s}\right)$ \\
\hline$Q$ & non-dimensional volume flow rate & & fluid density $\left(\mathrm{kg} / \mathrm{m}^{3}\right)$ \\
\hline $\mathrm{Re}$ & Reynolds number & & cylinder angular velocity (Rad/sec) \\
\hline
\end{tabular}

Various other pumping ideas were proposed to overcome the valve problem associated with positive displacement pumps. Electrohydrodynamic pumps utilize dielectric fluids, where the induced charges in the fluid will displace due to the moving electric field passing through it [5]. Electrokinetic pumps, similar to electrohydrodynamic pumps, use the moving electric field to displace the ions in the electric double layer of the electrolyte, rather than the charges in a dielectric fluid [6]. The liquid ions drag the rest of the fluid with it due to the viscosity, hence the fluid moves with nearly a uniform velocity profile. Sequential generation of thermal bubbles was also used to force liquids through microchannels [7]. A bubble is first introduced to function as a check valve to prevent the fluid from moving backward. Afterwards, another heater generates an additional bubble, which grows in the opposite direction of the first bubble, pumping the liquid in the desired direction. The process is then repeated by turning the heaters on and off in order to generate a continuous flow. To summarize, all the micropump concepts mentioned above, although applicable, are complex and involve the use of different auxiliary components in order to make the fluid environment suitable for pumping action to occur.

The viscous micropump, first introduced by Sen et al. [1], incorporates both applicability at the microscale and simplicity in design. The viscous micropump is simply a cylinder placed eccentrically inside a channel with its axis perpendicular to the channel axis. When the cylinder rotates, a net force is transferred to the fluid due to the unequal shear rates on the upper and lower surfaces of the cylinder, thus forcing the fluid to displace. Its operation depends mainly on viscous forces and can operate in any situation where viscous forces are dominant. This situation would exist for either low viscosity liquids in micro passages, due to the high surface-to-volume ratio characteristic of MEMS, or for highly viscous liquids, such as heavy polymers, in macro ducts. Sen et al. performed an experiment to test the pump performance. The study focused on the effect of the channel height, rotor eccentricity, and angular velocity on the pump performance and on the fluid bulk velocity in the duct.

In a later study, the same research team performed a numerical simulation of the viscous micropump solving Navier-Stokes equations for the case of the cylindrical 
rotor [8]. Critical values for optimum performance were calculated. The highest bulk velocity was achieved when the spacing between the plates is around one and half times the cylinder diameter for a fixed eccentricity, or at the maximum eccentricity for a fixed plates spacing. The load-flow rate curve for the pump was also plotted, with part of the curve in the negative part of the flow rate axis. This showed that the flow will change direction if the load is increased beyond the pump capability. The maximum efficiency of the pump was determined to be approximately $2.5 \%$ for the optimum plate spacing. However, it was observed that the viscous dissipation might cause a measurable temperature rise since viscous forces are the driving forces.

In order to check the effect of viscous dissipation on the pump performance, the same team performed a second numerical simulation [9]. In this study, they solved the continuity equation, the momentum equation with temperature-dependent viscosity, and the energy equation with viscous dissipation terms retained, all coupled together. It was determined that viscous dissipation would not cause a measurable rise in the bulk temperature of the fluid in MEMS applications, yet it may cause a significant rise in the fluid temperature combined with steep temperature gradients near the rotor where the shear stresses are maximum. Decourtye et al. [10] introduced three-dimensional effects of the sidewalls of the channel in their study. As expected, the pump performance decreased in terms of bulk velocity, yet pumping action existed even for channel widths less than the rotor diameter. It was observed that the sidewall effect reduces the channel height corresponding to maximum bulk velocity, and also increases the backpressure at which back flow occurs.

The present work will extend the previous studies mentioned above to account for the transient performance of the viscous micropump. The time required for the micropump to reach steady state, the time dependence of the drag and lift forces, and the viscous resisting torque on the cylinder will be computed. The pressure distribution on the cylinder surface will be also included in the study. As an indicator of the overall performance of the micropump, the efficiency will be reported for different geometries and operating conditions.

\section{PROBLEM DESCRIPTION}

\section{Pump Geometry}

The micropump is composed of a cylinder with diameter $d$, placed inside a channel of height $h$, with its axis perpendicular to the channel axis. Figure 1 shows a schematic

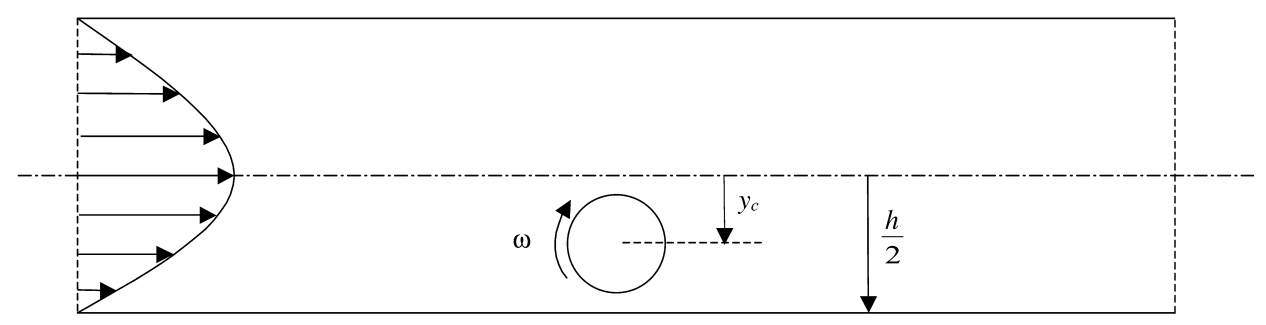

Figure 1. Schematic of the micropump geometry. 
of the pump geometry. The main geometrical parameters in the study will be the channel height $(S)$ defined as

$$
S=\frac{h}{d}
$$

and the cylinder eccentricity $\epsilon$, which indicates the cylinder position inside the channel. The eccentricity is defined as:

$$
\epsilon=\frac{y_{c}}{\frac{h}{2}-\frac{d}{2}}
$$

Based on this definition, $\epsilon=0$ corresponds to the cylinder being centered on the channel axis and $\epsilon=1$ corresponds to the cylinder touching the lower wall.

The cylinder is forced to rotate with an angular velocity $\omega$. In this problem, Reynolds number will be based on the cylinder surface velocity $U=\frac{\omega d}{2}$ since the average velocity in the channel $\bar{u}=\frac{1}{h} \int_{0}^{h} u d y$ is an output of the solution. Therefore, Reynolds number will be defined as:

$$
\operatorname{Re}=\frac{U d}{v}=\frac{\omega d^{2}}{2 v}
$$

The nondimensional average velocity inside the channel will be defined as:

$$
u^{*}=\frac{\bar{u}}{U}=\frac{\bar{u}}{\frac{\omega d}{2}}
$$

and the nondimensional flow rate will be defined as:

$$
Q=u^{*} \cdot S
$$

The pressure is specified on the inlet and outlet of the channel. A higher pressure is specified at the outlet to simulate the pressure head the pump should supply. This head is needed to overcome the pressure drop in whatever circuit the pump is attached to. The nondimensional pressure rise is defined as:

$$
\Delta P^{*}=\frac{P_{\text {out }}-P_{\text {in }}}{\frac{\rho v^{2}}{d^{2}}},
$$

where $P_{\text {out }}$ is the pressure on the pump outlet and $P_{\text {in }}$ is the pressure on the pump inlet, $\rho$ is the fluid density, and $v$ is the fluid kinematic viscosity.

The scale used to nondimensionalize the time in the simulation was chosen to be the time taken by the rotor to finish one complete revolution:

$$
t^{*}=\frac{t}{\frac{2 \pi}{\omega}}=\frac{t \omega}{2 \pi}
$$


According to this definition, the nondimensional time is simply the number of revolutions of the rotor. This provides an easier tracking of the changes in the flow field over time regardless of the rotor angular velocity.

The drag, lift, and moment coefficients are defined as

$$
\begin{aligned}
& C_{D}=\frac{F_{D}}{\frac{1}{2} \rho U^{2} D} \\
& C_{L}=\frac{F_{L}}{\frac{1}{2} \rho U^{2} D}
\end{aligned}
$$

and

$$
C_{M}=\frac{M}{\frac{1}{2} \rho U^{2} D^{2}}
$$

where $U$ is the cylinder surface velocity mentioned earlier.

\section{NUMERICAL SOLUTION}

The 2-D Navier-Stokes equations will be solved for the specified geometry where all the dimensions will be normalized by the cylinder diameter $d$. The main independent parameters in the solution will be the channel height $S$, the rotor eccentricity $\epsilon$, Reynolds number Re, and the pressure load $P^{*}$. The flow will be assumed laminar, incompressible, and unsteady, and the fluid itself is considered Newtonian with constant properties. Noslip, no-penetration boundary conditions are assumed on the microchannel walls and the cylinder surface. The pressure is specified on the inlet and outlet of the microchannel. Pressure will always be assumed to be zero gauge pressure at the inlet and its value at the exit will be varied to simulate different loads. The fluid will be assumed to be initially at rest and the motion will start from rest by rotating the cylinder clockwise with an angular velocity $\omega$.

The CFD package FLUENT 6.0 is used to solve the Navier-Stokes equations numerically. This CFD package uses the finite volume method and supports unstructured grids. It enables the use of different discretization schemes and solution algorithms, together with various types of boundary conditions. As part of the same package, a preprocessor, Gambit, is used to generate the required grid for the solver. Different meshes were used at the beginning to determine the optimum grid size and to ensure grid-independent solution, and results are presented in Table 1. The last grid size presented in Table 1 was used in this study and is an unstructured grid with triangular elements. The cylinder surface was divided into 100 equally spaced elements. The upper and lower walls were divided into 200 nonuniformly spaced elements using the Bell shaped meshing scheme. The grid was finer in regions near the center and adjacent to the cylinder, and was coarser in regions far upstream and downstream. The Bell shaped scheme meshes the edge so that the node distribution follows a normal distribution curve centered at the geometric center of the edge. In addition to this meshing method, which refines the grid in the cylinder region, grid adaptation by the solver itself was performed in the gap 
Table 1. Properties of different grids used and comparison of results

\begin{tabular}{ccccc}
\hline \multirow{2}{*}{$\begin{array}{c}\text { Number of cylinder } \\
\text { elements } \times \text { number } \\
\text { of wall elements }\end{array}$} & \multicolumn{2}{c}{$\begin{array}{c}\text { Number of } \\
\text { control volumes }\end{array}$} & & \begin{tabular}{c}
$u^{*}$ \\
\cline { 2 - 3 }
\end{tabular} \\
\cline { 2 - 4 } & $S=1.5$ & $S=2.5$ & $(S=1.5, \epsilon=0.6)$ & $(S=2.5, \epsilon=0.95)$ \\
\hline $10 \times 50$ & 589 & 985 & 0.0527 & 0.0632 \\
$20 \times 100$ & 2241 & 3652 & 0.0703 & 0.0859 \\
$40 \times 150$ & 5151 & 8611 & 0.0757 & 0.0919 \\
$80 \times 150$ & 6341 & 11533 & 0.077 & 0.0932 \\
$100 \times 200$ & 11044 & 18983 & 0.0769 & 0.0943 \\
\hline
\end{tabular}

between the cylinder and the lower wall for cases of high eccentricities, where this gap size is very small.

The Piso-Simple algorithm, PISO standing for Pressure-Implicit with Splitting of Operators, was used for the pressure-velocity coupling. It is nearly the same as the SIMPLE algorithm, presented in [11], except that it takes into account two additional corrections, which are the neighbor correction and the skewness correction [12]. The PISO algorithm requires less time than the SIMPLE algorithm, rendering it more suitable for transient applications. Two different discretization schemes were used for the time and momentum equations. A power law scheme was used for the momentum equation while a second order discretization scheme was used for the time derivatives. Under-relaxation was used during the solution with the under-relaxation factors varying between 0.3 and 1 to ensure convergence.

As a convergence criterion, the solver iterated the equations until the scaled residuals were less than $10^{-5}$ or until it stabilized at a constant value, which is still small enough to ensure convergence. This value varied approximately from $10^{-5}$ to $3 \times 10^{-4}$, based on the parameters for each specific case. The change of the average velocity inside the micropump channel with time was used as a criterion to determine the optimum step size. The results for $\Delta t=0.0001 \mathrm{~s}, 0.001 \mathrm{~s}$, and $0.01 \mathrm{~s}$ were nearly the same, while for $\Delta t=0.1 \mathrm{~s}$, instability and large fluctuations in the average velocity occur. The time step chosen for nearly all the cases studied was $0.001 \mathrm{~s}$, which was small enough to recognize all the changes in the flow field over time, and, in addition, was large enough to achieve reasonable computation time.

\section{RESULTS AND DISCUSSION}

Before the transient operation of the micropump was studied, some steady-state cases were simulated and the results were compared with existing numerical and experimental results. Existing experimental results for steady-state cases were obtained from Sen et al. [1], while numerical results were obtained from Sharatchandra et al. [8]. The effect of the micropump channel height on the pump flow rate was studied experimentally and numerically in the two references mentioned above respectively. Figure 2 shows that the results obtained in the present study are in very good agreement with both the computational and experimental results. It should be noted that the definition of the eccentricity $\epsilon$ is different in the present work than that in the references mentioned above, where the eccentricity, $\epsilon=0.9$, in the present study corresponds to the value of the maximum ec- 


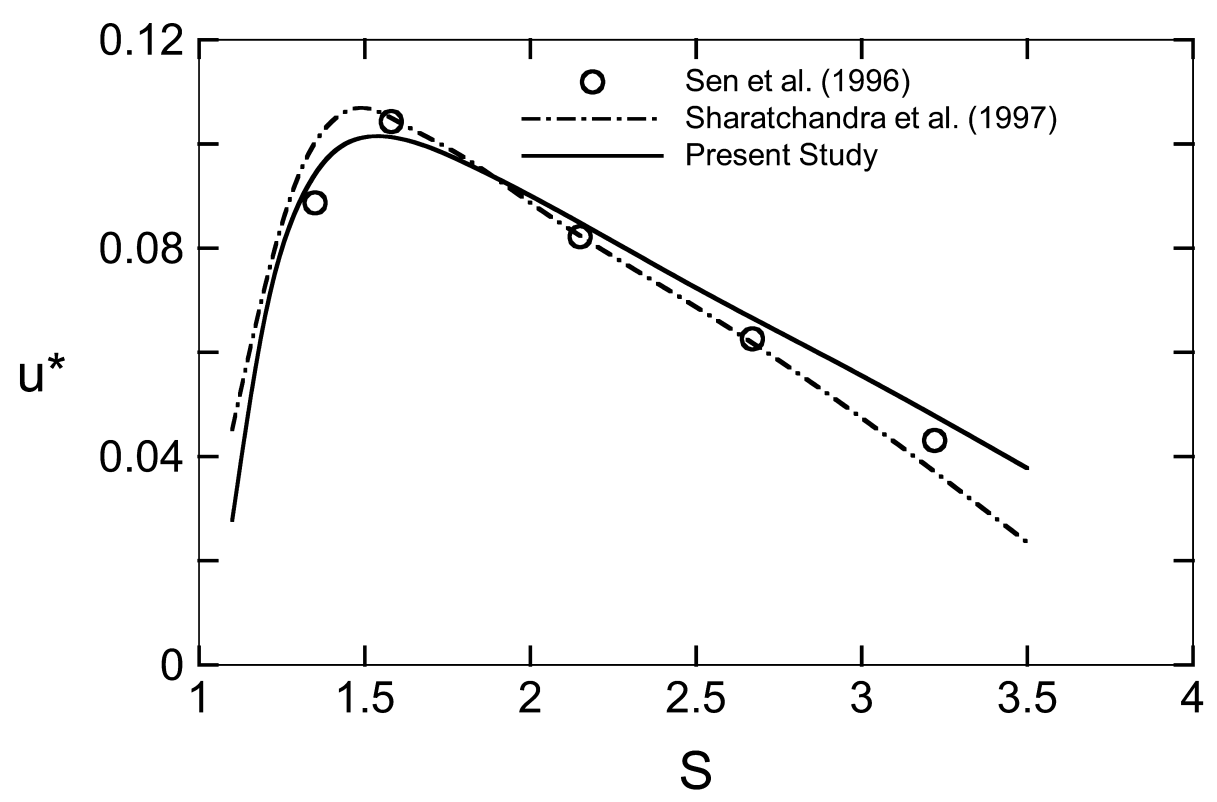

Figure 2. Comparison of average velocity vs. channel height at $\operatorname{Re}=0.5, \Delta P^{*}=0.5$, and $\epsilon=0.9$.

centricity, $\epsilon_{\max }$, in the two references mentioned above. Figure 3 compares the variation of average velocity with rotor eccentricities obtained by Sharatchandra et al. [8] with that obtained in the present study, while Figure 4 plots the nondimensional volumetric flow rate versus the nondimensional pressure $(\mathrm{Q}-\mathrm{P}$ curve) for Shratchandra et al. [8] and the present study. The results in both figures are in good agreement with each other.

\section{Effect of Microchannel Height}

The pump performance was found to change considerably when the channel height was changed from $S=1.5$ to $S=3.5$. Figure 5 shows the effect of the channel height $S$ on the start-up performance of the micropump. It is clear that when the channel height is increased, the pump requires a longer time to reach the steady-state flow rate. This is due to the fact that, for cases of higher $S$, the momentum of the fluid layers adjacent to the cylinder is diffused through a larger number of fluid layers in order to reach the region adjacent to the upper wall, which takes a longer time depending on the viscosity of the working fluid. The micropump time constant was defined as the time taken by the pump from the instant it starts until the instant the average velocity inside the pump channel reaches $99 \%$ of its steady-state value at the same conditions. This time constant was found to increase with increasing the channel height as shown in Figure 6.

Figure 7 shows the development of the flow field with time inside the micropump. At the beginning, the vortices start from the upper surface of the cylinder, Figure 7a, and then move upward with time, Figures $7 \mathrm{~b}$ and $7 \mathrm{c}$, until they separate from the cylinder surface to form either a pair of vortices, as in the cases of low $S$ values, Figure 7d, or one big vortex, as in the cases for high values of $S$. Figure 8 gives the pressure 


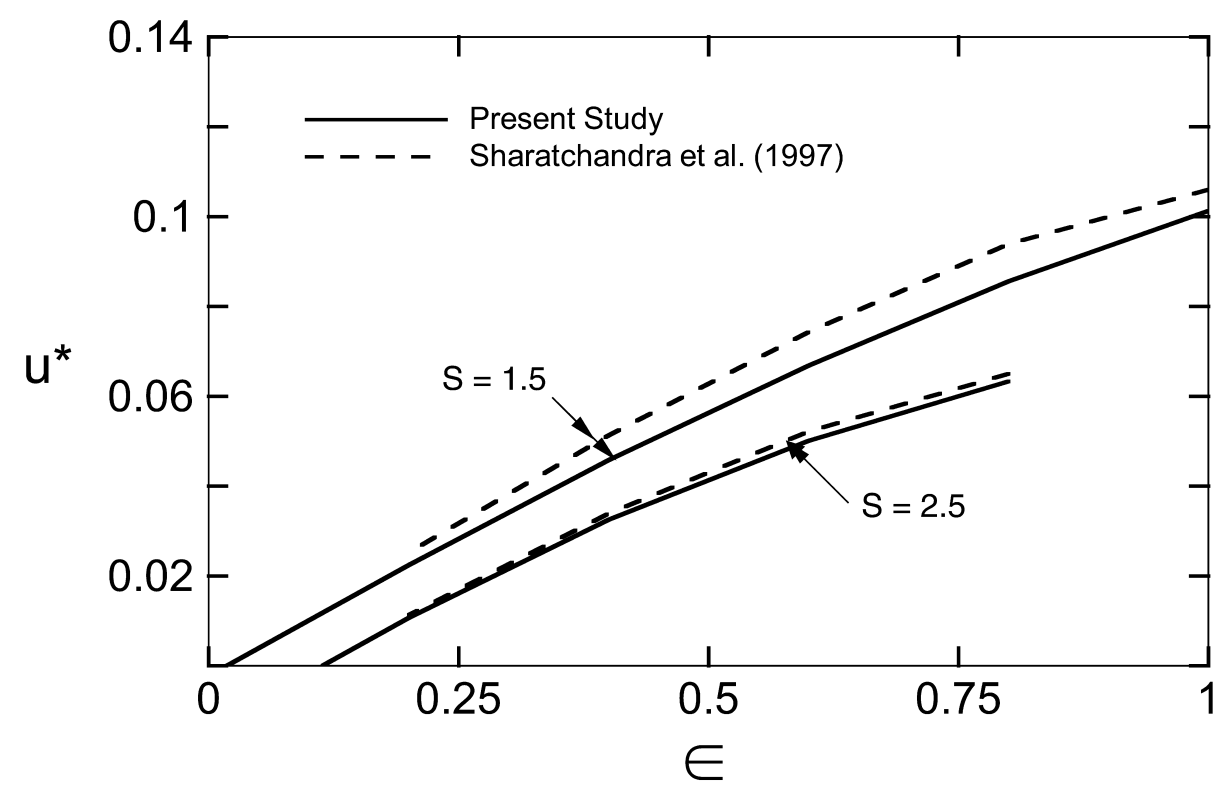

Figure 3. Comparison of average velocity vs. eccentricity at $\Delta P^{*}=1$ and $\operatorname{Re}=1$.

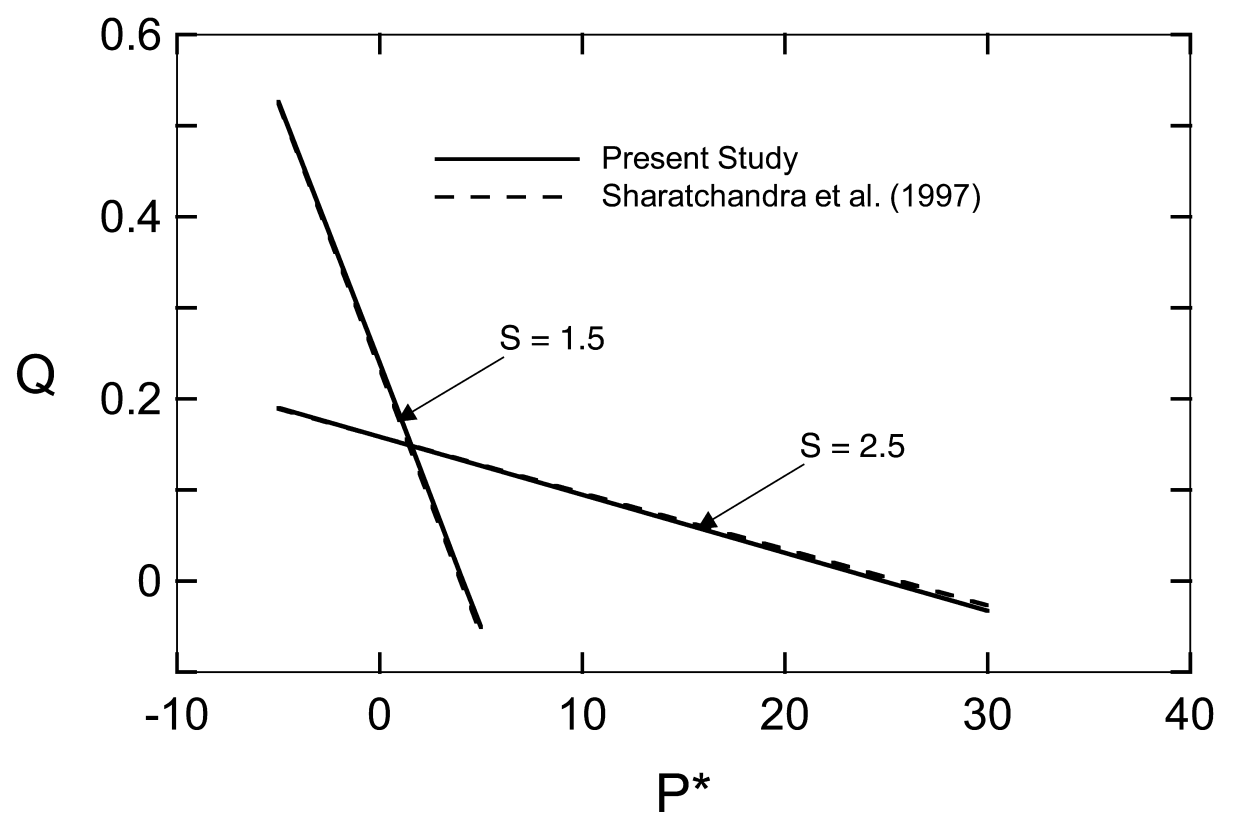

Figure 4. Flow rate vs. pump load at $\operatorname{Re}=0.5$ and $\epsilon=0.9$. 


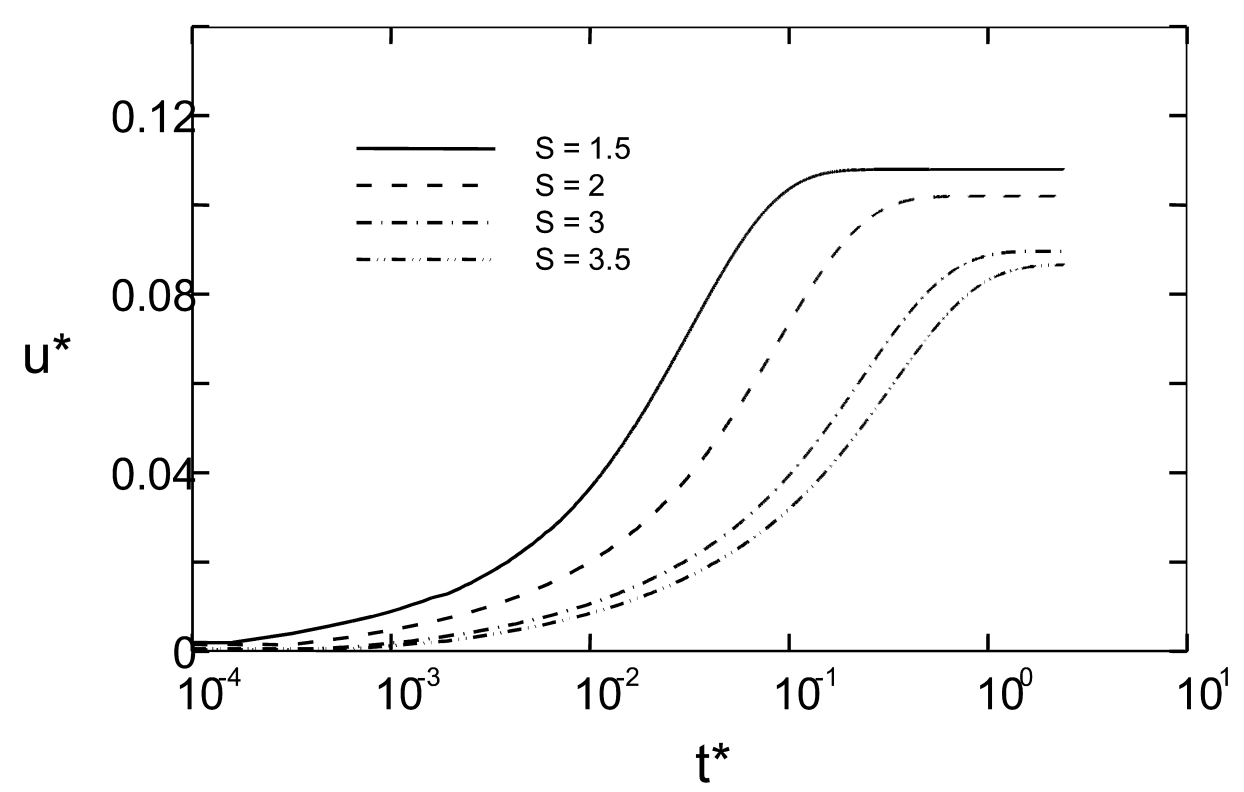

Figure 5. Start-up operation for different channel heights at $\epsilon=0.95, \Delta P^{*}=0$, and $\operatorname{Re}=1$.

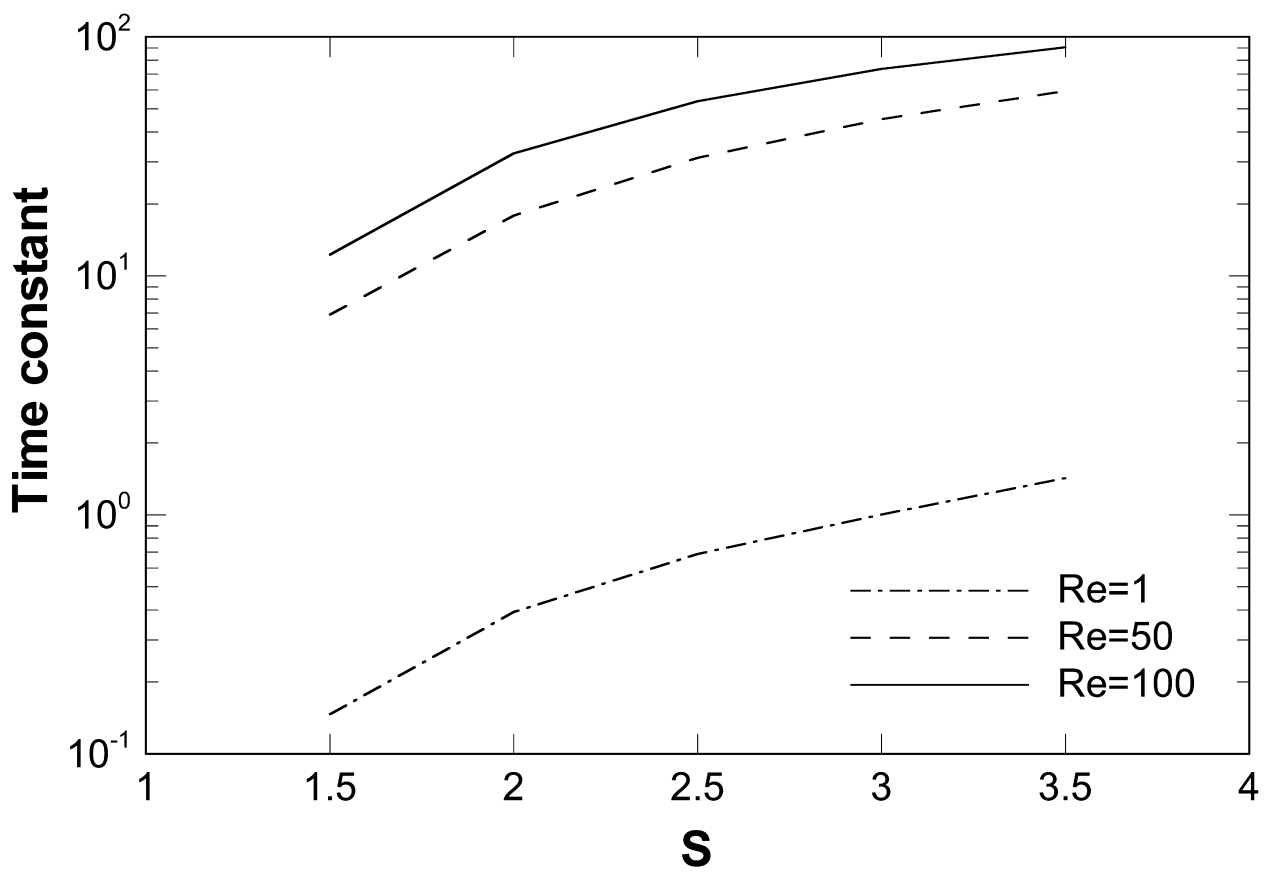

Figure 6. Time constant as a function of channel height at different $\operatorname{Re}\left(\epsilon=0.95, P^{*}=0\right)$. 


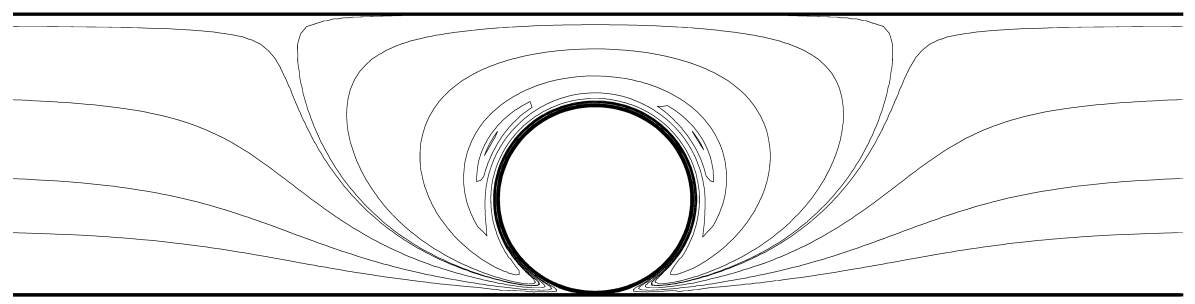

(a) $t^{*}=0.48 \mathrm{e}-3$

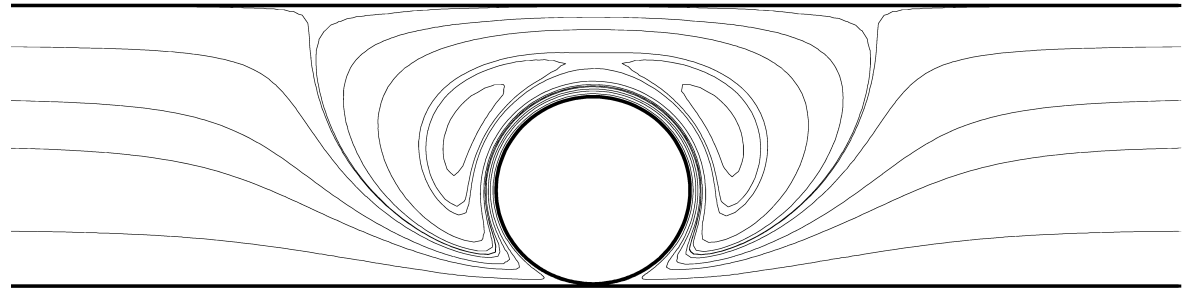

(b) $\mathrm{t}^{*}=4.8 \mathrm{e}-3$

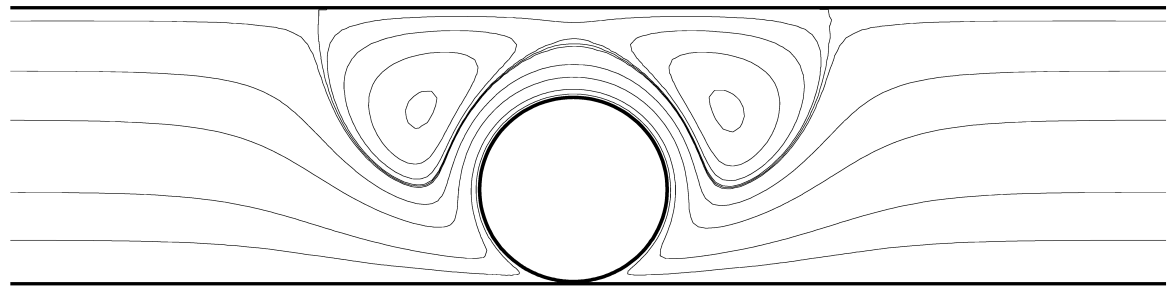

(c) $t^{*}=31.98 \mathrm{e}-3$

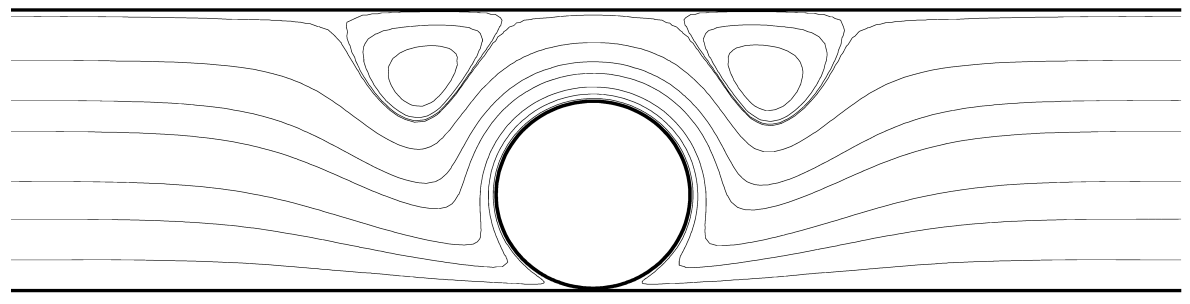

(d) $t^{*}=$ Steady State

Figure 7. Flow pattern development with time at $S=1.5, \epsilon=0.95, \Delta P^{*}=0$, and $\operatorname{Re}=1$. 


\begin{tabular}{|ll|}
\hline 11 & 0.00889765 \\
\hline 9 & 0.00155044 \\
\hline 7 & $-4.45893 \mathrm{E}-05$ \\
\hline 5 & -0.00167854 \\
\hline 3 & -0.0058941 \\
\hline 1 & -0.0103316 \\
\hline
\end{tabular}

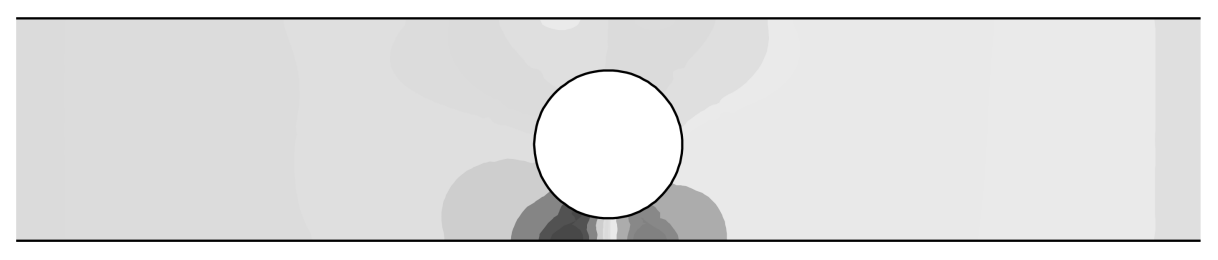

Figure 8. Steady-state pressure contours around the cylinder at $S=1.5, \epsilon=0.4, \Delta P^{*}=0$, and $\operatorname{Re}=1$.

distribution around the cylinder in the viscous micropump. There are two distinct zones when it comes to the pressure distribution. The first one is the high-pressure zone on the downstream lower cylinder surface. The high-pressure zone results from the presence of the lower wall, which obstructs the vertical component of the fluid velocity creating a semi-stagnation zone. The low-pressure zone on the upstream lower cylinder surface results from the suction created by the vertical component of the fluid velocity, which is directed away from the lower wall.

The variation of the drag, lift, and moment coefficients with time are shown in Figure 9. The drag coefficient was found to decrease with increasing the channel height, as shown in Figure 9a. When channel height $S$ increases, the lower gap is bigger and the effect of the lower wall obstructing the vertical component of the velocity is much less than when the lower gap is small. This causes a reduction in the horizontal component of the pressure force on the cylinder and consequently a reduction in the drag coefficient. In addition, there is a reduction in the shear rate on the upper cylinder surface as the size of the gap between the cylinder and the upper wall increases.

The lift coefficient is dependent on the channel height and was found to be one order of magnitude lower than the drag coefficient, Figure $9 \mathrm{~b}$. The large difference between the values of the drag and lift coefficients is due to the nature of pressure distribution around the cylinder as shown in Figure 8. Since there are high- and low-pressure zones below the cylinder, and since the pressure distribution on the upper cylinder surface is of moderate value, it is clear that the upward force resulting from the high-pressure zone below the cylinder will be balanced by the downward force resulting from the pressure difference between the lowest pressure zone and the pressure zone on the opposite cylinder surface. The lift force is, thus, the result of the viscous forces on the cylinder surface. It should be mentioned here that the flow field around the cylinder is not symmetrical and this is very clear in cases of high Reynolds number where the downstream vortex grows in size while the upstream vortex diminishes. The lift coefficient was found to decrease with increasing channel height $S$ because the velocity gradient on the upper cylinder surface decreases with increasing the channel height and thus decreasing the lift coefficient. 


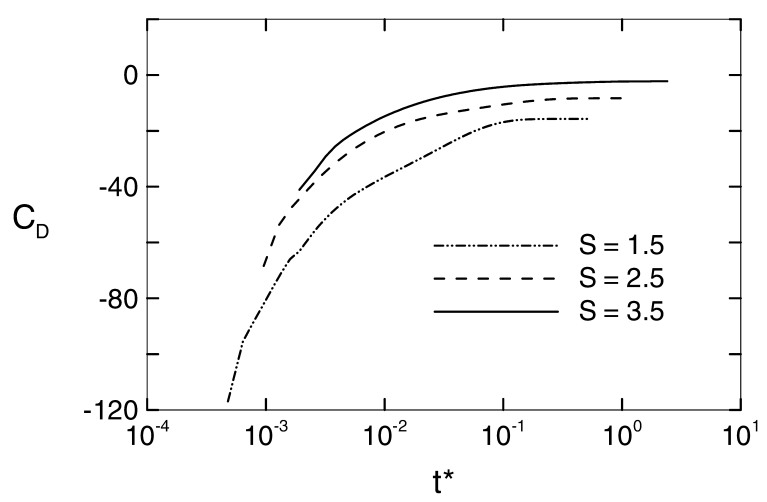

(a)

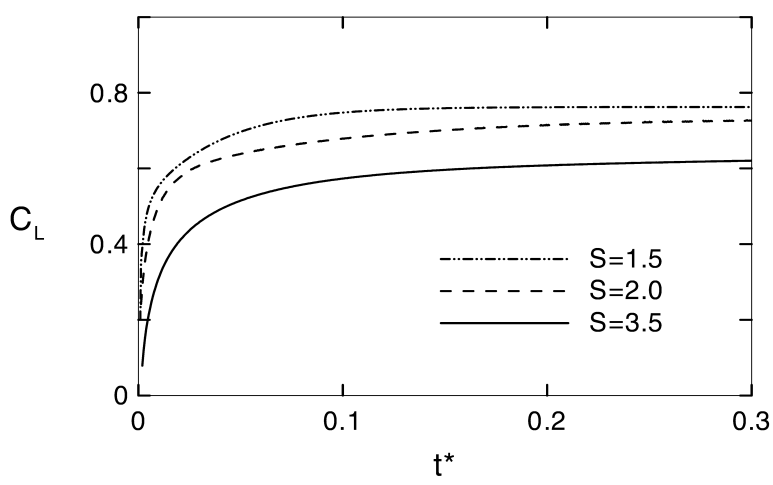

(b)

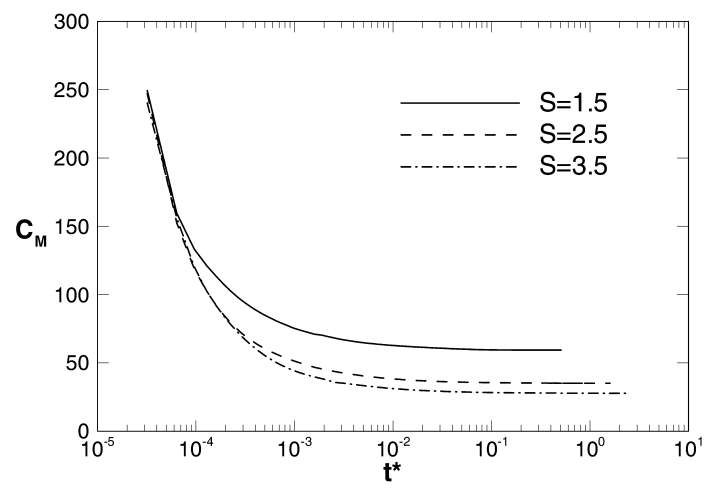

(c)

Figure 9. Variation of drag, lift, and moment coefficients with time at $\epsilon=0.95, \Delta P^{*}=0$, and $\operatorname{Re}=1$. 
The resisting torque on the cylinder surface is due to the viscous forces since the pressure forces are perpendicular to the cylinder surface. At the beginning of the cylinder rotation the moment coefficient is highest, Figure 9c, because the fluid layer adjacent to the cylinder surface is moving with the cylinder velocity, while the fluid layers adjacent to that layer are nearly stationary, which increases the resisting torque. With time, momentum is diffused through the fluid layers away from the cylinder and the velocity gradient decreases, causing a reduction in the shear stress and resisting torque on the cylinder surface, producing a lower moment coefficient.

In order to determine how efficient the micropump is, the efficiency was defined and the effect of the flow parameters on its value was investigated. The net energy addition to the flow is only in the form of pressure rise (flow energy) at the pump outlet, since the kinetic energy at the inlet and exit are equal. Accordingly, the micropump efficiency can be defined as:

$$
\eta=\frac{\text { Flow energy rise }}{\text { Input mechanical energy }}
$$

Therefore, the micropump efficiency may be obtained from:

$$
\eta=\frac{\frac{m \Delta P}{\rho}}{M \omega}
$$

In nondimensional terms, this reduces to:

$$
\eta=\frac{\Delta P^{*} u^{*} S}{C_{m} \operatorname{Re}^{2}}
$$

Figure 10 shows how the efficiency $\eta$ varies with time for different channel heights. It was determined that increasing the channel height reduces the pumping efficiency since the velocity gradients on the cylinder surface, and hence the viscous forces, i.e., pumping forces, decrease.

\section{Effect of Rotor Eccentricity}

Figure 11 shows the different start-up curves at different eccentricities. It is clear that when the eccentricity increases the steady-state average velocity inside the channel increases and the micropump requires more time to reach this steady-state operation. This is due to the fact that increasing the cylinder eccentricity increases the mass flow rate inside the channel directly and thus more time is needed for the viscous forces between the fluid layers to overcome the inertia of the fluid to attain this higher velocity.

Figure 12 shows how the drag and moment coefficients vary with time at various eccentricities. The steady-state drag coefficient, Figure 12a, is always negative, and increases with an increase in the cylinder eccentricity. At low eccentricities, the drag coefficient is initially a small value, after which it increases with time, and then finally decreases and returns to its steady-state value. For high eccentricities, the drag coefficient is initially a large value and gradually decreases to its steady-state value. Figure $12 \mathrm{~b}$ shows the variation of the moment coefficient with time for different eccentricities, where the moment coefficient increases with increasing eccentricity. This is due to the 


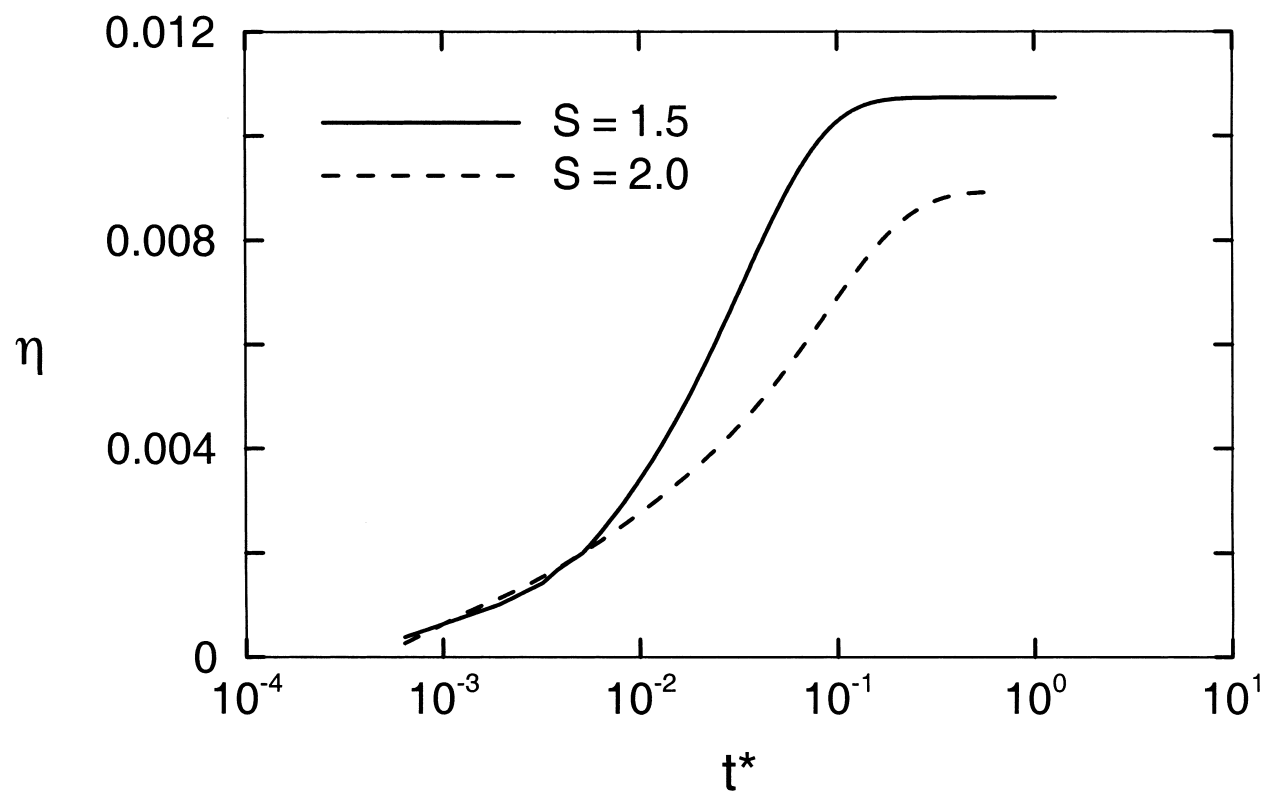

Figure 10. Variation of micropump efficiency with time for different channel heights at $\epsilon=0.95, \Delta P^{*}=5$, and $\operatorname{Re}=1$.

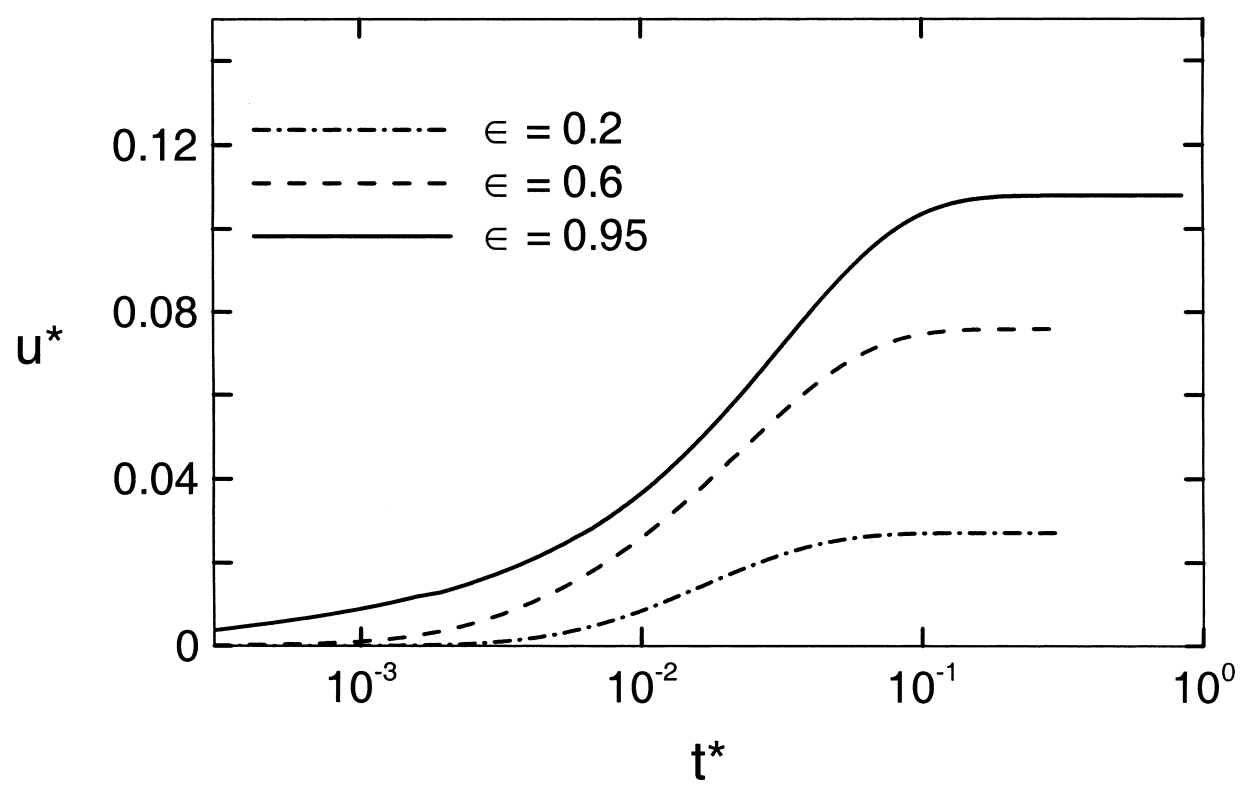

Figure 11. Variation of average velocity with time for different eccentricities at $S=1.5, \Delta P^{*}=0$, and $\operatorname{Re}=1$. 


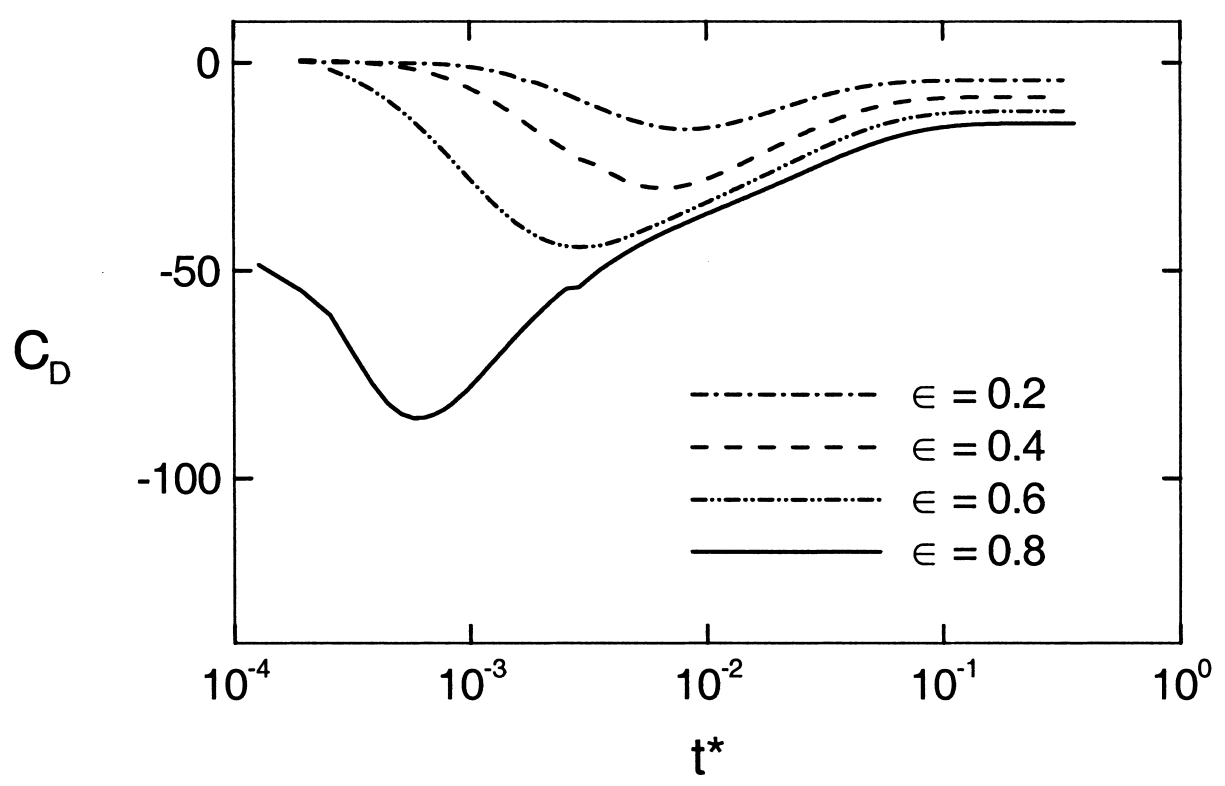

(a)

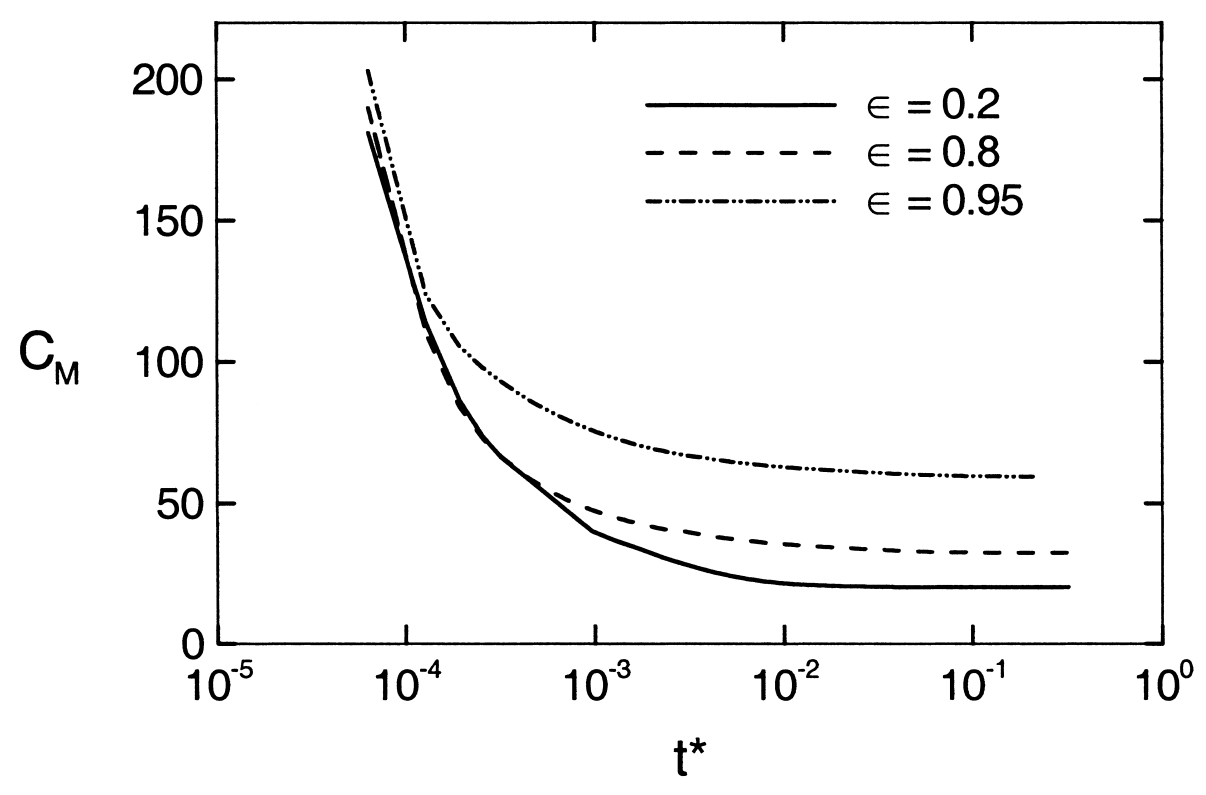

(b)

Figure 12. Time variation for $S=1.5, \Delta P^{*}=0$, and $\operatorname{Re}=1$ at different eccentricities of a) the drag coefficient and b) the moment coefficient. 


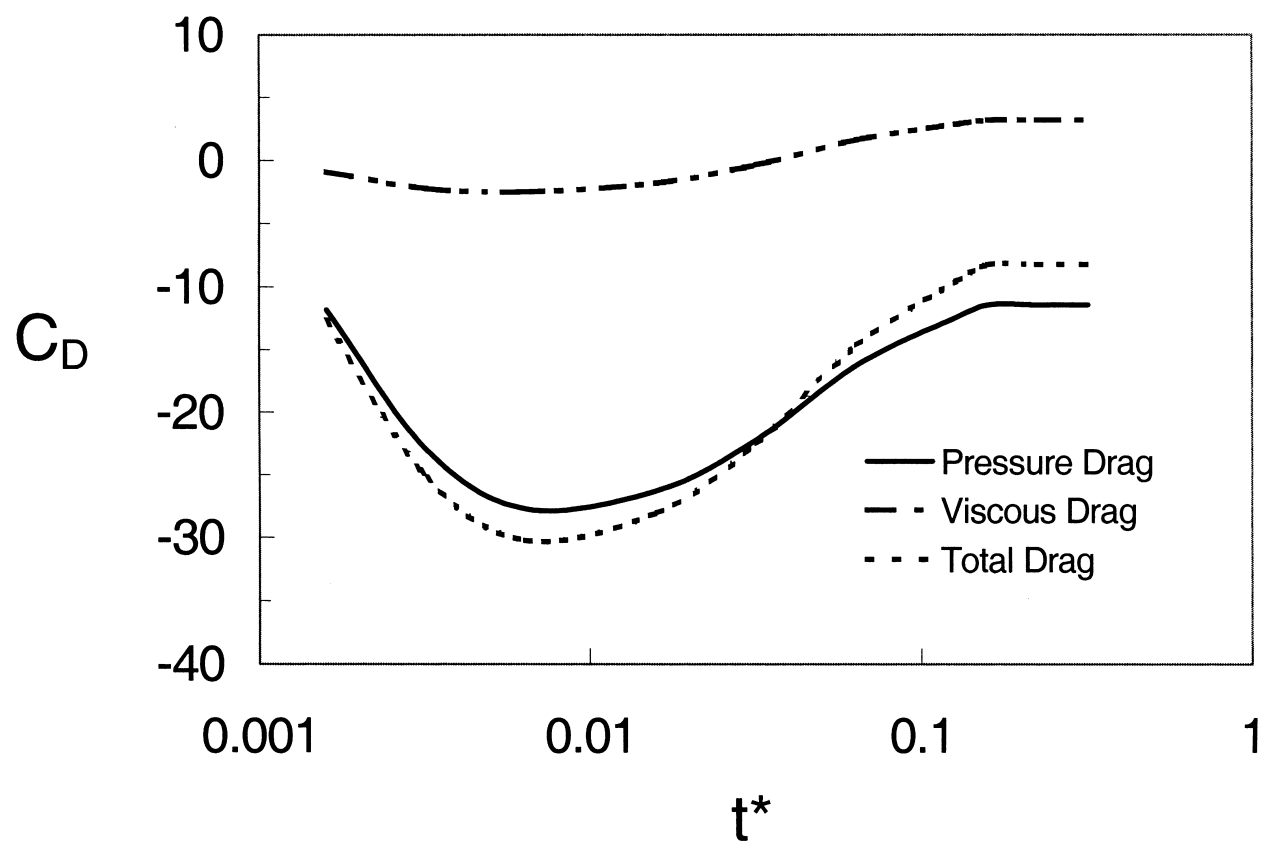

Figure 13. Viscous, pressure, and total drag coefficients for $\epsilon=0.4$ at $S=1.5, \Delta P^{*}=0$, and $\operatorname{Re}=1$.

reduction in the gap size between the cylinder and the lower wall, resulting in an increase in the shear stress on the cylinder surface.

To account for why the drag coefficient increases and then decreases with time, as shown in Figure 12a, the viscous and pressure drag must be considered. Figure 13 shows the variation of the pressure and viscous drags with time at $\epsilon=0.4$. At low eccentricities, the pressure in the semi-stagnation zone behind the cylinder increases with time until it reaches a maximum value, and then afterwards decreases slightly. At the beginning, the fluid motion is restricted to the vicinity of the cylinder and the lower wall obstructs the vertical component of the fluid velocity, raising the pressure and forming the semi-stagnation zone. With time, and due to this high-pressure zone, the fluid's velocity increases and is redirected away from this zone, consequently reducing its pressure. At high eccentricities, the lower gap between the cylinder and the lower wall is so small that the obstruction of the lower wall causes the pressure to reach its maximum very rapidly. The pressure then decreases with time due to the velocity development in the semi-stagnation zone. The pressure drag naturally follows the same trend of the pressure.

Figure 14 shows that the dependence of the efficiency on the cylinder eccentricity has a maximum. According to Eq. (12), the efficiency is proportional to $u^{*}$ and inversely proportional to $C_{M}$. For low eccentricities, the average velocity $u^{*}$ is small, so the efficiency should be small. However, the moment coefficient $C_{M}$ is also small due to the low velocity gradient on the cylinder surface, which tends to increase the efficiency. For the cases of high eccentricities, the situation is reversed. The average velocity $u^{*}$ is high, which tends to increase the efficiency, but the moment coefficient $C_{M}$ is also high, which tends to decrease the efficiency. As a result, the efficiency is expected to have its highest 


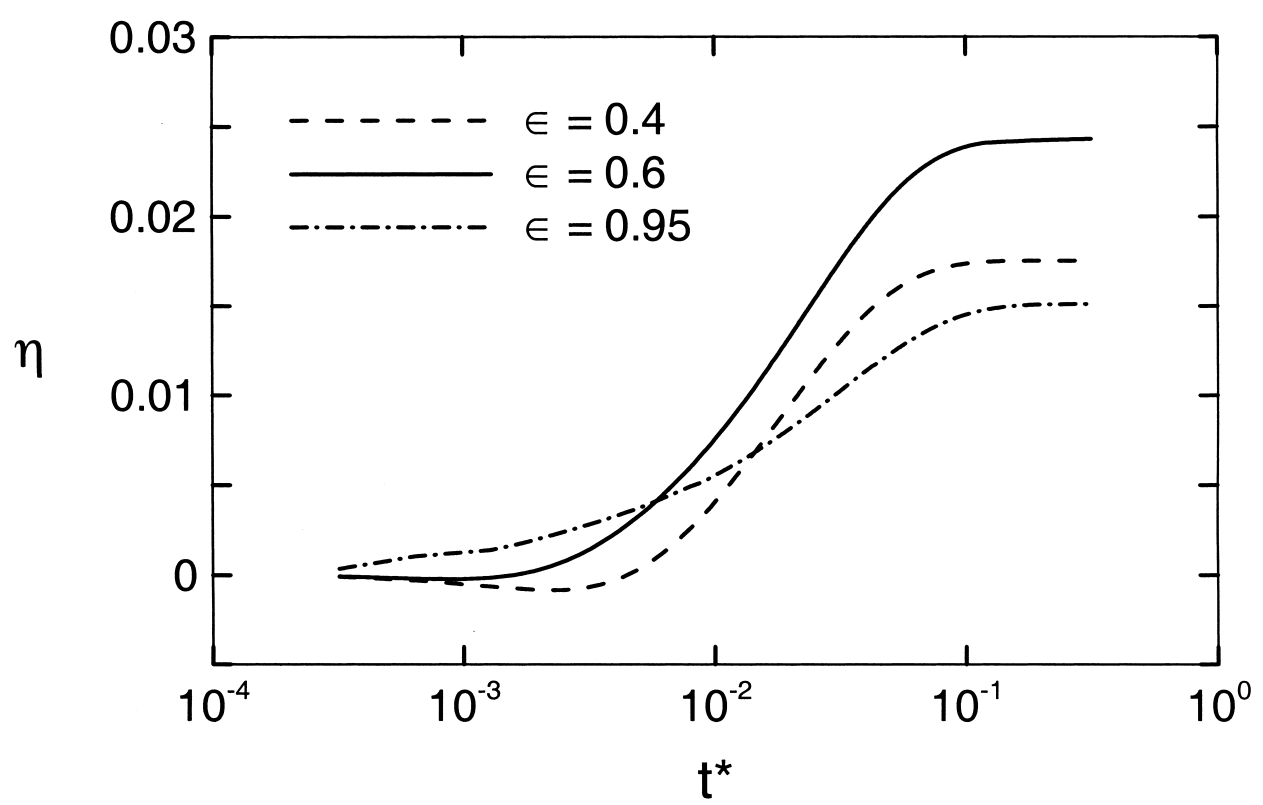

Figure 14. Variation of micropump efficiency with time for different eccentricities at $S=1.5, \Delta P^{*}=10$, and $\operatorname{Re}=1$.

value at moderately high cylinder eccentricities where the average velocity is high and the moment coefficient is still relatively small. This is confirmed by Figure 15, which shows the variation of the efficiency with the backpressure at different eccentricities.

\section{Effect of Reynolds Number and Pump Load}

Figure 16 shows the variation of the velocity with time at different Reynolds numbers. The nondimensional velocity is normalized by the cylinder surface velocity, which increases linearly with increasing Reynolds number. Hence, the actual fluid velocity is much higher even though the nondimensional velocity for higher Reynolds number is less than that for low Reynolds number. The starting time for the three curves is different because the nondimensional time is calculated using Eq. (7). Thus, the nondimensional time will be larger even if the time step is smaller for cases of high Reynolds number, due to the large rotational velocity of the cylinder. It should be noted that when Reynolds number is increased, the upstream vortex decreases in size until it disappears completely at very high Reynolds numbers. The drag, lift, and moment coefficients were found to decrease with increasing Reynolds number. This is due to the increase in the cylinder surface velocity, which is used for normalizing the drag and lift force. The lift and moment coefficients decreased with increasing Reynolds number due to the decrease of the viscous forces relative to the inertia forces.

The pump load is modeled by increasing the pressure on the outlet boundary of the pump to simulate the pressure rise needed from the pump to overcome the pressure losses in the external fluid circuit. Figure 17 shows the change of the average velocity inside the micropump with time at different pump loads. It is clear that the pump average velocity 


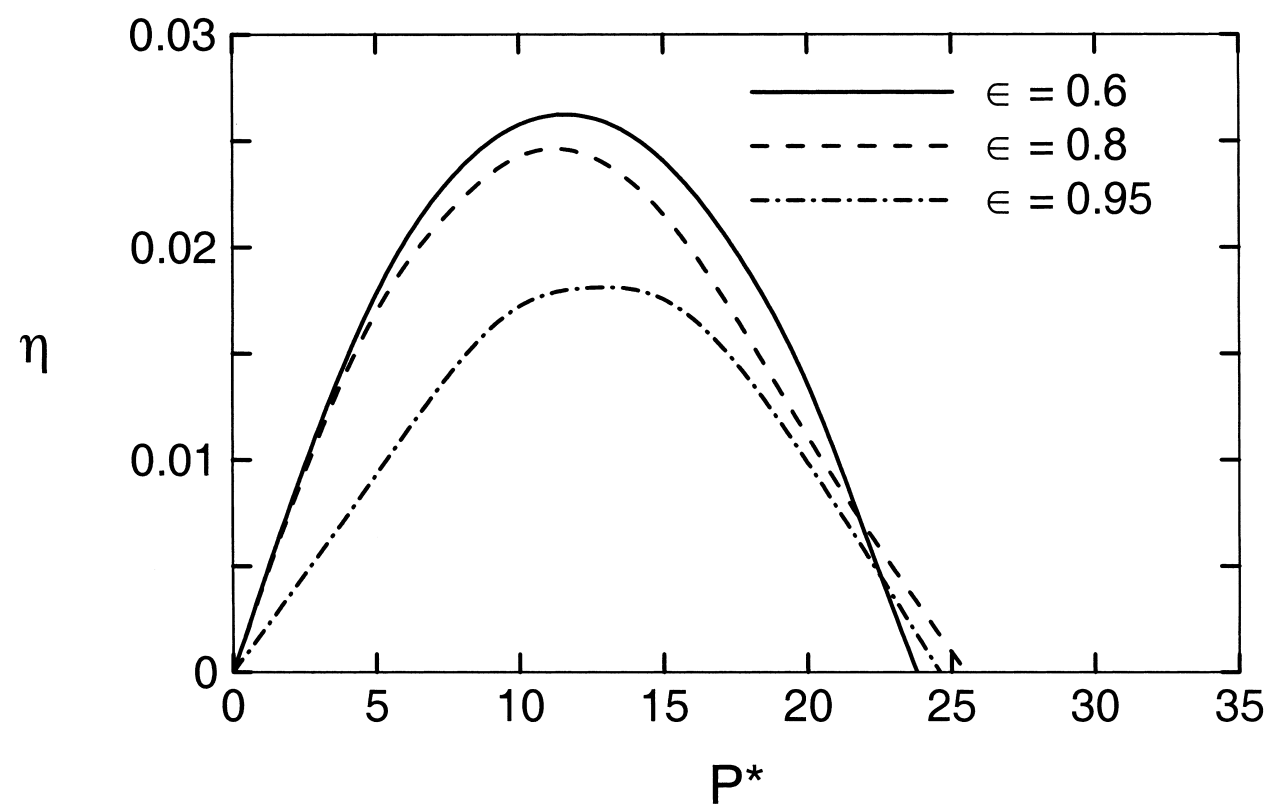

Figure 15. Efficiency as a function of pressure for different eccentricities at $S=1.5$ and $\operatorname{Re}=1$.

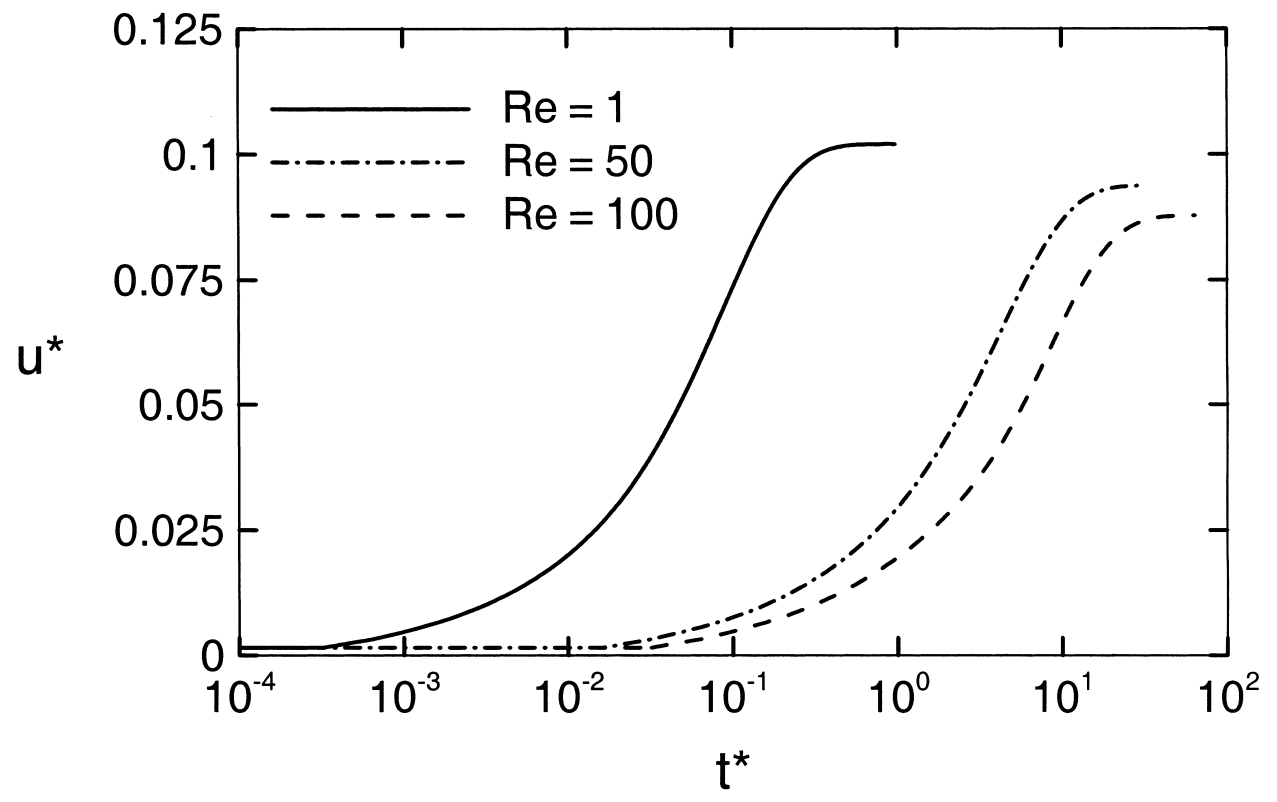

Figure 16. Variation of average velocity with time for different Reynolds numbers at $S=1.5, \epsilon=0.95$, and $\Delta P^{*}=0$. 


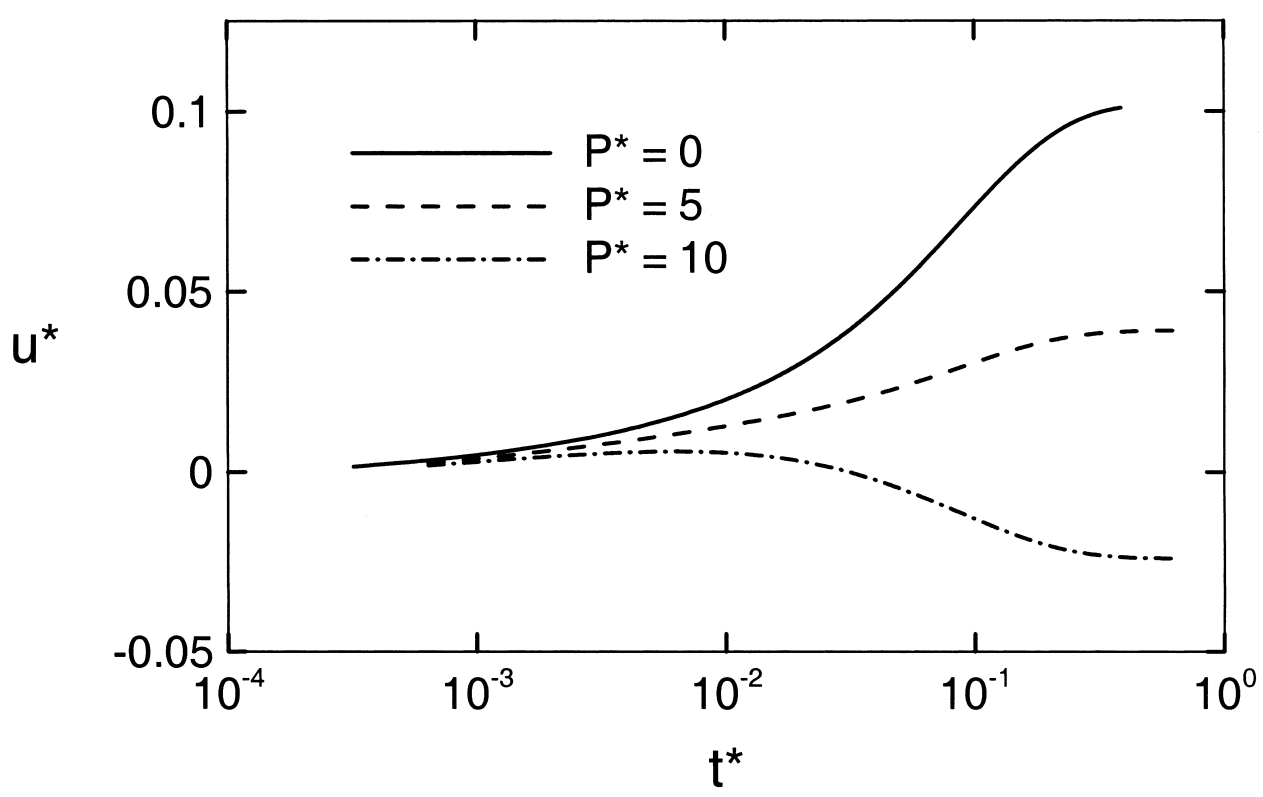

Figure 17. Variation of average velocity with time for different pressures at $S=2, \epsilon=0.95$, and $\operatorname{Re}=1$.

decreases with increasing pump load. When the pressure exceeds the maximum load the pump can deliver, a backflow occurs and the average velocity becomes negative, as is the case for the third curve " $P^{*}=10$." It is important to note that, in cases of reversed flow, the fluid passes through the space between the upper wall and the vortices above the cylinder instead of passing through the space between the upper cylinder surface and the vortices. Figure 18 shows how the pumping efficiency rises to its steady-state value corresponding to the applied backpressure. The best operating conditions for the pump will be at moderate pressures, which achieve both a high-energy addition rate per fluid element and a relatively high overall flow rate. Figure 15 provides confirmation of this fact.

\section{CONCLUSIONS}

In the present work, the transient performance of the viscous micropump was investigated numerically. The steady-state results obtained were in very good agreement with previously reported experimental results. The effect of varying the geometrical parameters, such as the channel height and cylinder eccentricity, as well as flow parameters, such as the cylinder angular velocity and the backpressure, on the pumping performance with time were studied separately. The time required for the pump to reach steady-state operation was calculated, and was found to depend significantly on the Reynolds number, channel height, and cylinder eccentricity. Reynolds number affected the time constant the most as it increased the time constant by two orders of magnitude when Reynolds number was varied from $\operatorname{Re}=1$ to $\operatorname{Re}=100$.

The rotor eccentricity was by far the most important parameter in determining the overall pump transient performance. Higher eccentricities were able to generate high backpressures, yet the pumping efficiency was lower than that for cases of relatively lower 


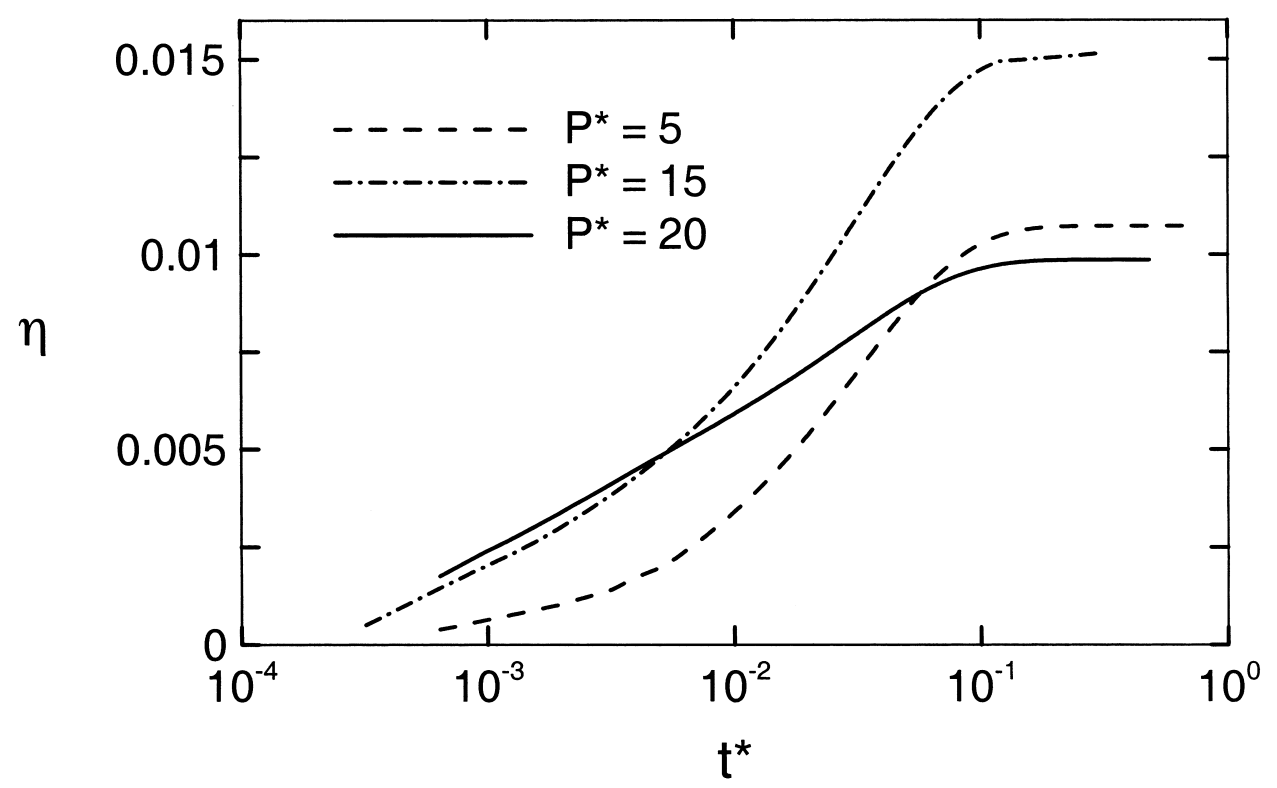

Figure 18. Variation of micropump efficiency with time at different pressures at $S=1.5, \epsilon=0.95$, and $\operatorname{Re}=1$.

eccentricities. Depending on the value of the backpressure and cylinder eccentricity, the pump startup in some cases was characterized by a backflow followed by a positive flow, or vice versa.

The development of the drag, lift, and moment coefficients was studied for different geometrical and flow conditions. The drag coefficient was always negative as expected and the pressure contribution to the drag force was always higher than the viscous force contribution, sometimes one order of magnitude higher. The lift coefficient is much smaller than the drag coefficient. The moment coefficient always decreased with time due to the flow field development around the cylinder, which reduced the velocity gradient, and hence the shear stress, on the cylinder surface over time.

All the micropump calculations are reported in nondimensional quantities, which allows for the prediction of the micropump performance, regardless of the dimensions or the fluid that is used. The viscous micropump can generate pressures ranging between $0.1 \mathrm{~Pa}$ up to $1 \mathrm{kPa}$ or more, with an efficiency ranging between $2 \%$ to $3 \%$, depending on the micropump dimensions and the fluid properties. The average velocity of the fluid inside the micropump reached up to $10 \%$ of the cylinder surface velocity, which, together with the channel height, determines the pump average flow rate.

In conclusion, the concept of the viscous micropump needs to be further studied in order to improve the efficiency and increase the pressure rise. The steady-state and transient operation of the pump and the parameters that affect them have now been studied. Other geometries that may possess superior viscous driving characteristics need to be suggested and studied. The simplicity of the viscous micropump design and its size flexibility provide great potential for this device in commercial applications and is thus worthy of further study in the future. 


\section{REFERENCES}

1. M. Sen, D. Wajerski, and M. Gad-el-Hak, A Novel Pump for MEMS Applications, Journal of Fluids Engineering, vol. 118, pp. 624-627, 1996.

2. P. Gravesen, J. Branebjerg, and O. S. Jensen, Microfluidics-A Review, Journal of Micromechanics and Microengineering, vol. 3, pp. 168-182, 1993.

3. E. Stemme and G. Stemme, Valveless Diffuser/Nozzle-Based Fluid Pump, Sensors and Actuators, A: Physical, vol. 39, pp. 159-167, 1993.

4. S. Erik and S. Goran, Valveless Diffuser/Nozzle-Based Fluid Pump, Sensors and Actuators, A: Physical, vol. 39, pp. 159-167, 1993.

5. S. F. Bart, L. S. Tavrow, M. Mehregany, and J. H. Lang, Microfabricated Electrohydrodynamic Pumps, Sensors and Actuators A: Physical, vol. 21, pp. 193-197, 1990.

6. D. J. Harrison, A. Manz, and P. G. Glavina, Electroosmotic Pumping within a Chemical Sensor System Integrated on Silicon, Proceedings of the International Conference on SolidState Sensors and Actuators Transducers, pp. 792-795, 1991.

7. C. J. Kim, Microfluidics Using the Surface Tension Force in Microscale, Microfluidic Devices and Systems III, Proceedings of the SPIE, vol. 4177, pp. 49-55, 2000.

8. M. C. Sharatchandra, M. Sen, and M. Gad-el-Hak, Navier-Stokes Simulation of a Novel Viscous Pump, Transaction of the ASME, Journal of Fluids Engineering, vol. 119, pp. 372-382, 1997.

9. M. C. Sharatchandra, M. Sen, and M. Gad-el-Hak, Thermal Aspects of a Novel Viscous Pump, Journal of Heat Transfer, vol. 120, pp. 99-107, 1998.

10. D. Decourtye, M. Sen, and M. Gad-el-Hak, Analysis of Viscous Micropumps and Microturbines, International Journal of CFD, vol. 10, pp. 13-25, 1998.

11. S. V. Patankar, Numerical Heat Transfer and Fluid Flow, Washington: Hemisphere Pub. Corp.; New York: McGraw-Hill, 1980.

12. FLUENT 6.0 user guide manual, Fluent, Inc., NH 2002. 